\title{
Mechanisms Of Response and Resistance to AML Therapies
}

\author{
Bilyana Stoilova ${ }^{1}$, Rachel Moore ${ }^{1}$, Marlen Metzner ${ }^{1}$, Zhihong Zheng ${ }^{2}$, Marina Konopleva ${ }^{2}$, \\ Courtney DiNardo ${ }^{2}$, , Paresh Vyas ${ }^{1}$ \\ ${ }^{1}$ MRC Molecular Haematology Unit, Weatherall Institute of Molecular Medicine, Radcliffe Department of Medicine, \\ University of Oxford. \\ ${ }^{2}$ Department of Leukemia, Divison of Cancer Medicine, MD Anderson Cancer Centre University of Texas, Houston \\ Texas.
}

*Corresponding author: paresh.vyas@imm.ox.ac.uk 750-1,500 words

\section{Keywords}

Single cell analysis; clonal hierarchy; genetic analysis; transcriptional analysis.

\section{Introduction}

Until recently prognostic and predictive biomarkers of response and resistance to blood cancer therapy have relied on analysis of populations of cells. Though we have learnt a lot from these studies, the field has now incorporated analysis at a single cell level. This is important as when cancers are exposed to therapy the unit of selection is the (single) cell. Single cell studies allow determination of the complex clonal structure of disease, in any one patient, providing information on coacquisition of genetic and epigenetic changes and their impact on the transcriptional and differentiation state of the cell, including differentiation arrest in the haemopoietic hierarchy.

We have used single cell analysis to study sequential patient samples, pre- and post-therapy with targeted agents and combination chemotherapy to understand the mechanisms of response and resistance in Acute Myeloid Leukaemia.

\section{Results and Discussion}

Our first study examined the clonal basis response and resistance to patients who received chemotherapy and allogeneic stem cell transplant (allo-SCT $)^{[1]}$. This showed that that in some patients there was clonal selection between diagnosis and allo-SCT with chemotherapy. In some relapsing patients there was also further clonal selection post allo-SCT. This study showed the importance of studying sequential samples and how different treatments can impose different selective pressures.

We next studied the clonal basis of response and resistance to single agent enasidenib in patients with relapsed/refractory IDH2 mutant $\mathrm{AML}^{[2]}$. In our workflow we initially determined the recurrent driver AML mutations by either exome sequencing or extensive targeted re-sequencing. We then determined the clonal structure through haematopoietic differentiation by single cell FACS-sorting of immunophenotyping leukaemic stem/progenitor cells, precursor cells and mature cells and then performing either genotyping or combined genotyping and transcriptome analysis ${ }^{[3]}$. To study the clonal and molecular basis of response we compared samples from trial entry with those in complete remission. To understand the mechanisms of resistance we compared sequential samples from diagnosis, remission and relapse.

Prior to treatment, our data show that there are complex clonal patterns of differentiation arrest. In some cases it was not possible to predict the clones that showed the most profound differentiation arrest. In those patients who achieved complete remission, the mechanism of response was a reversal of differentiation arrest. In most cases leukaemic and preleukaemic clones differentiated to give mature blood cells. In rare cases, haemopoiesis was restored by normal cells. One explanation for this is that leukaemic and preleukaemic clones were more likely to terminally differentiate and had reduced self- 
renewal. However, as patients are not cured with single agent enasidenib, leukaemic cells must persist. Our data also showed that there are multiple mechanisms of relapse including emergence of clones with IDH1 mutation, mutations in signaling molecules (RAS mutations), monosomy 7 , and mutations in transcription factors. In summary, in relapsed/refractory IDH1 AML the clonal complexity of disease is such that relapse is inevitable by multiple mechanisms.

We have now extended these studies to patients treated with azacitidine alone, enasidenib combined with azacitidine, Ivosidenib combined with venetoclax, single magrolimab and finally patients treated with curative intent with combination chemotherapy. We will present the outcome of those studies.

\section{References}

1. Quek, L., P. Ferguson, M. Metzner, I. Ahmed, A. Kennedy, C. Garnett, S. Jeffries, C. Walter, K. Piechocki, A. Timbs, R. Danby, M. Raghavan, A. Peniket, M. Griffiths, A. Bacon, J. Ward, K. Wheatley, P. Vyas, and C. Craddock, Mutational Analysis of Disease Relapse in Patients Allografted for Acute Myeloid Leukemia. Blood Advances, 2016. 1: p. 193204.

2. Quek, L., M.D. David, A. Kennedy, M. Metzner, M. Amatangelo, A. Shih, B. Stoilova, C. Quivoron, M. Heiblig, C. Willekens, V. Saada, S. Alsafadi, M.S. Vijayabaskar, A. Peniket, O.A. Bernard, S. Agresta, K. Yen, K. MacBeth, E. Stein, G.S. Vassiliou, R. Levine, S. De Botton, A. Thakurta, V. Penard-Lacronique, and P. Vyas, Clonal heterogeneity of acute myeloid leukemia treated with the IDH2 inhibitor enasidenib. Nat Med, 2018. 24(8): p. 1167-1177.

3. Rodriguez-Meira, A., G. Buck, S.A. Clark, B.J. Povinelli, V. Alcolea, E. Louka, S. McGowan, A. Hamblin, N. Sousos, N. Barkas, A. Giustacchini, B. Psaila, S.E.W. Jacobsen, S. Thongjuea, and A.J. Mead, Unravelling Intratumoral Heterogeneity through High-Sensitivity Single-Cell Mutational Analysis and Parallel RNA Sequencing. Mol Cell, 2019. 73(6): p. 1292-1305 e8. 